\section{BRAZIULIAN JOURNAL \\ OF MEDICAL AND BIOLOGICAL RESEARCH}

www.bjournal.com.br
ISSN 1414-431X

Volume 45 (12) 1102-1340 December 2012

\section{BIOMIDICAL SCIENCES}

AND

CLINICAL INVESTIGATION

Braz J Med Biol Res, December 2012, Volume 45(12) 1287-1294

doi: $10.1590 / \mathrm{S} 0100-879 \mathrm{X} 2012007500147$

Pediatric pain: prevalence, assessment, and management in a teaching hospital

M.B.M. Linhares, F.N.P. Doca, F.E. Martinez, A.P.P. Carlotti, R.G.M. Cassiano, L.I. Pfeifer, C.A. Funayama, L.R.G. Rossi and G.A. Finley

The Brazilian Journal of Medical and Biological Research is partially financed by

\section{욛NPq}

da Ciência e Tecnologia

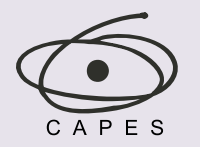

Ministério da Educação

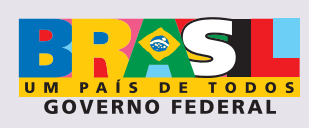

DTFAPESP

Institutional Sponsors

๑ SHIMADZu

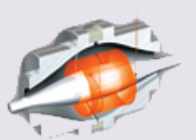

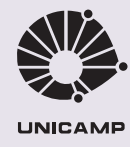
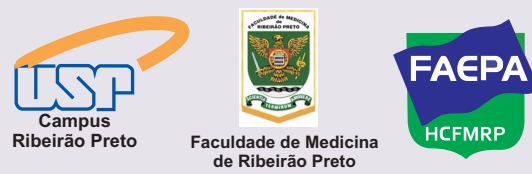

UNICAMP

lore High - Performance MS Orbitrap Technology In Proteomics \& Metabolomics analitica Thermo 


\title{
Pediatric pain: prevalence, assessment, and management in a teaching hospital
}

\author{
M.B.M. Linhares ${ }^{1}$, F.N.P. Doca1,5, F.E. Martinez ${ }^{2}$, A.P.P. Carlotti ${ }^{2}$, \\ R.G.M. Cassiano ${ }^{1}$, L.I. Pfeifer ${ }^{1}$, C.A. Funayama ${ }^{1}$, L.R.G. Rossi ${ }^{3}$ and G.A. Finley ${ }^{4}$ \\ ${ }^{1}$ Departamento de Neurociências e Ciências do Comportamento, Faculdade de Medicina de Ribeirão Preto, \\ Universidade de São Paulo, Ribeirão Preto, SP, Brasil \\ 2Departamento de Puericultura e Pediatria, Faculdade de Medicina de Ribeirão Preto, \\ Universidade de São Paulo, Ribeirão Preto, SP, Brasil \\ ${ }^{3}$ Divisão de Enfermagem, Hospital das Clínicas, Faculdade de Medicina de Ribeirão Preto, \\ Universidade de São Paulo, Ribeirão Preto, SP, Brasil \\ ${ }^{4}$ Department of Anesthesia, Center for Pediatric Pain Research, Dalhousie University, NS, Canada \\ ${ }^{5}$ Programa de Pós-Graduação em Processos de Desenvolvimento Humano e Saúde, \\ Instituto de Psicologia, Universidade de Brasília, Brasília, DF, Brasil
}

\begin{abstract}
The goal of this study was to examine the prevalence, assessment and management of pediatric pain in a public teaching hospital. The study sample consisted of 121 inpatients (70 infants, 36 children, and 15 adolescents), their families, 40 physicians, and 43 nurses. All participants were interviewed except infants and children who could not communicate due to their clinical status. The interview included open-ended questions concerning the inpatients' pain symptoms during the $24 \mathrm{~h}$ preceding data collection, as well as pain assessment and pharmacological/non-pharmacological management of pain. The data were obtained from $100 \%$ of the eligible inpatients. Thirty-four children/adolescents $(28 \%)$ answered the questionnaire and for the other $72 \%$ (unable to communicate), the family/health professional caregivers reported pain. Among these 34 persons, 20 children/ adolescents reported pain, $68 \%$ of whom reported that they received pharmacological intervention for pain relief. Eighty-two family caregivers were available on the day of data collection. Of these, 40 family caregivers (49\%) had observed their child's pain response. In addition, $74 \%$ reported that the inpatients received pharmacological management. Physicians reported that only $38 \%$ of the inpatients exhibited pain signs, which were predominantly acute pain detected during clinical procedures. They reported that $66 \%$ of patients received pharmacological intervention. The nurses reported pain signs in $50 \%$ of the inpatients, which were detected during clinical procedures. The nurses reported that pain was managed in $78 \%$ of inpatients by using pharmacological and/or non-pharmacological interventions. The findings provide evidence of the high prevalence of pain in pediatric inpatients and the under-recognition of pain by health professionals.
\end{abstract}

Key words: Pain; Inpatients; Policy; Clinical audit

\section{Introduction}

Pain is a stressful experience that is considered to be a global health problem, and children are the most vulnerable and under-served population. Despite the exponential increase in scientific evidence about pediatric pain in the last few decades, there are many barriers to the transfer of knowledge to clinical practice. Consequently, children still experience unnecessary pain during hospitalization (1-4).

Audit studies have been useful for the systematic analysis of pain assessment and management in general hospitals (5) and pediatric hospitals $(3,4)$. In one report, a prospective, cross-sectional survey was conducted in a Canadian pediatric teaching hospital in order to identify the prevalence and intensity of pain and the frequency of medical chart notation of pain assessment and pharmacological management (3). The data collection was performed on a single, typical day in the hospital, and a structured questionnaire was used to interview the inpatients or their caregivers. The results showed that pediatric pain was under-assessed and under-treated. Consequently, actions were taken to improve the management of pediatric pain in

Correspondence: M.B.M. Linhares, Departamento de Neurociências e Ciências do Comportamento, FMRP, USP, Av. Tenente Catão Roxo, 2650, Salas 52/53, 14048-900 Ribeirão Preto, SP, Brasil. Fax: +55-16-3602-4504. E-mail: linhares@fmrp.usp.br

Received March 27, 2012. Accepted August 31, 2012. Available online September 21, 2012. Published December 17, 2012. 
the hospital, including educational initiatives for all nurses and pediatricians and a new pain management clinical guideline. Similar results were obtained by Stevens et al. (4) in a study using a different methodology focused on medical chart analysis.

These studies highlight the strengths and weaknesses of pain management in the context of a given institution and establish baseline data to compare with future studies that repeat the audits. Policies for pain relief and management are necessary for sustainable change in institutional settings. The ChildKind International Initiative is a program for the reduction of childhood pain and has been endorsed by major international health organizations (6). A pain audit survey conducted in the hospital is the first step in fulfilling the ChildKind criteria.

To our knowledge, no pain audits have been conducted in Brazilian hospitals. Other studies have detected problems related to pediatric pain, including: a) disagreement among children's caregivers about the intensity of the pediatric pain (7); b) physicians' and nurses' limited understanding of pain assessment tools and the available methods for treatment of pediatric pain, which results in pain under-treatment $(8,9)$; c) health professionals' lack of knowledge and misconceptions concerning analgesic and opioid prescriptions (10); d) inadequate pharmacological management of pediatric pain (11), and e) the high incidence of medical errors associated with pain medication prescriptions (12).

The aim of the present study was to examine the pediatric pain prevalence and to characterize the practice of pain assessment, and its non-pharmacological and pharmacological management, in a public teaching hospital setting.

\section{Material and Methods}

\section{Setting and study sample}

The study was carried out at Hospital das Clínicas, Faculdade de Medicina de Ribeirão Preto, Universidade de São Paulo (HC-FMRP-USP, Ribeirão Preto, SP, Brazil). This is a tertiary public teaching hospital providing high complexity level care in the Brazilian Federal Health System. The hospital has 170 beds for pediatric patients from birth to 18 years of age, including the University Campus Unit (122 beds) and the Emergency Unit (48 beds). This hospital was selected as a pilot site for the ChildKind Initiative (6). The eligible sample for the study included all children and adolescents hospitalized in the pediatric services on the target day for data collection, as well as their family caregivers who were available on that day and the nurses and physicians responsible for the care of the children.

The present study was approved by the Ethics Committee Board of the hospital and informed consent was obtained from the children's legal guardians and the participants.

\section{Instruments}

Four brief questionnaires were developed by expert researchers on pediatric pain for each participant category (see Supplementary material for Interview guides). These questionnaires were intended to screen for the prevalence of pain and to identify the pain assessment procedures and the non-pharmacological and pharmacological pain management regimens. Additionally, a data collection sheet was elaborated for the medical/nurse chart analysis (see Supplementary material for Medical/Nurse chart analysis).

\section{Procedure}

The procedure used in the present study was based on the principles of the ChildKind International Initiative (13). Data collection was performed at the end of 2009 on three typical days in the hospital. On the first and second days, data were collected at the University Campus Unit. On the third day, data were collected at the pediatric services of the Emergency Unit. The participants did not have prior knowledge of the data collection in order to control for potential biases due to changes in practice.

A team of 26 investigators (psychologists and occupational therapists) was trained to carry out the data collection. The appropriate questionnaires were used for individual face-to-face interviews. Children older than 2 years were interviewed in order to obtain a self-report of pain. The interviewers were allocated to services that were different from their usual affiliations in the hospital in order to assure that they were blind to patient status. At the beginning of each interview, demographic data were obtained for the participants. For all participants, the main focus of the interview was the pain experienced/observed in the pediatric inpatients in the $24-h$ period preceding the data collection day. In addition, the medical/nursing charts on the day of the interview were reviewed by three skilled pediatric physicians and by two pediatric psychologists.

\section{Data analysis}

First, the open questions were classified into thematic categories to perform a systematic categorical analysis. The categories were as follows: a) presence/absence of pain, b) pain localization, c) pain descriptors, d) context of pain, e) type of clinical procedure, f) presence/absence of pain assessment, g) type of pain assessment procedure, h) presence/absence of pain management, and i) type of pharmacological and nonpharmacological management. In addition, the written records in the medical/nurse charts were categorized by the presence/ absence of pain and types of assessment, management, and medications prescribed, as well as whether they were effectively administered to the inpatients.

Second, all data were organized into a database for statistical analysis using the Statistical Package for Social Sciences $^{\circledR}$ (SPSS, version 19.0). Data are reported as frequency and percentage, or median and range, and the association between categorical variables was analyzed using the chi-square test. Association analyses were performed between patients' self-reports of pain or their 
family-caregivers' pain reports and the following two variables: health professionals' perceptions of pain (physicians/ nurses) and pain records in the medical charts, focusing on prevalence, assessment, and management of pediatric pain of 86 cases for which we had all information available. The level of statistical significance established in the study was $5 \%(P \leq 0.05)$.

\section{Results}

\section{Characteristics of the study sample}

Of 170 beds available in the Pediatric Services, 121 were occupied on the target days for data collection. Data were obtained for all inpatients. All family caregivers who were present in the hospital on the target day were also interviewed $(\mathrm{N}=82)$.

Forty physicians answered questionnaires concerning pain of 115 inpatients. Physician interview data could not be obtained for six cases because four physicians refused to participate in the study and two physicians were not available to answer the questionnaire. Forty-three nurses answered questions concerning pain of 120 inpatients. Nursing interview data could not be obtained for one case.

Table 1 shows that 94 patients $(77 \%)$ were hospitalized in the University Campus Unit and 27 patients (23\%) in the Emergency Unit. In the University Campus, the majority of patients $(29 \%)$ were hospitalized in the Pediatric Clinical Ward including the specialties of Oncology, Gastroenterology, and Pneumology. Twenty-six per cent of patients were from the Neonatology Service (Intermediate Special Nursery and Neonatal Intensive Care Unit). The remaining inpatients were from the Pediatric Neurology Ward and other Clinical and Surgery Services (Epilepsy, Pediatrics, Neurosurgery, Orthopedics, Cardiology, and Urology). In the Emergency Unit, the inpatients were predominantly from the Pediatric Clinical Ward and the Pediatric Intensive Care Unit.

The study population ( $N=121$ inpatients) included 20 neonates (17\%), 50 infants (41\%), 19 preschool aged children (16\%), 17 school-aged children (14\%), and 15 adolescents (12\%). Seventy-two were males (60\%) and 49 females $(40 \%)$. The median length of stay in the hospital was 9 days (range $=1-631$ days), excluding 2 outliers who were hospitalized for more than 2 years. Patients were hospitalized for clinical reasons (68\%), surgical reasons (17\%), diagnostic investigation (14\%), and social reasons (1\%).

The physicians' profiles showed that $55 \%$ were females, and most of them were between 24 and 30 years of age (85\%). Seventy-eight percent were young physicians with 3 years of professional experience. The majority of nurses were females (95\%), and $61 \%$ of them had more than 10 years of professional experience.

\section{Pediatric pain reports: children/adolescents and family caregivers}

The data were obtained from $100 \%$ of the 121 inpa- tients hospitalized on the target day. Thirty-four children/ adolescents $(28 \%)$ answered the questionnaire and for $72 \%$ inpatients $(\mathrm{N}=86)$, the family caregivers or the health professionals reported their pain signs because the patients were unable to self-report pain (70 neonates/infants and 16 children with clinical problems). Only one child refused to participate in the study.

Of the 34 children/adolescents $(28 \%)$ who were able to answer the interview, 20 inpatients (59\%) complained of pain in the $24 \mathrm{~h}$ preceding the target day for data collection. Of the 20 children/adolescents who self-reported their pain, $90 \%$ communicated the presence of pain in the last $24 \mathrm{~h}$, primarily to the family caregivers or to the nursing staff.

Eight-two family caregivers were available in the hospital during data collection and answered questions about their children's pain. Of these, $89 \%$ were mothers, $4 \%$ were fathers, $4 \%$ were grandmothers, and $3 \%$ were other family

Table 1. Distribution of the inpatients in the Emergency Unit and University Campus Unit/Pediatric Services of the University Hospital, Faculty of Medicine at Ribeirão Preto/USP $(\mathrm{N}=121)$.

\begin{tabular}{|c|c|c|}
\hline Units/Pediatric services & Frequency & Percent \\
\hline \multicolumn{3}{|l|}{ Emergency Unit } \\
\hline Pediatric clinical ward & 14 & 12 \\
\hline Pediatric intensive care unit & 7 & 6 \\
\hline Infectious disease ward & 4 & 3 \\
\hline Burn unit & 2 & 2 \\
\hline \multicolumn{3}{|l|}{ University Campus Unit } \\
\hline \multicolumn{3}{|l|}{ Neonatology service } \\
\hline Neonatal intensive care unit & 14 & 12 \\
\hline Intermediate special nursery & 18 & 14 \\
\hline Pediatric intensive care unit & 7 & 6 \\
\hline \multicolumn{3}{|l|}{ Pediatric clinical ward } \\
\hline Oncology & 7 & 6 \\
\hline Gastroenterology & 5 & 4 \\
\hline Pneumology & 4 & 3 \\
\hline Cardiology & 3 & 2 \\
\hline Nephrology & 3 & 2 \\
\hline Infectious diseases & 3 & 2 \\
\hline Endocrinology & 2 & 2 \\
\hline Immunology & 1 & 1 \\
\hline Rheumatology & 1 & 1 \\
\hline Not specified & 7 & 6 \\
\hline Pediatric neurology, clinical & 5 & 4 \\
\hline Epilepsy surgery & 2 & 2 \\
\hline Pediatric surgery & 3 & 2 \\
\hline Neurosurgery & 1 & 1 \\
\hline Orthopedic surgery & 4 & 3 \\
\hline Cardiology surgery & 2 & 2 \\
\hline Urology surgery & 2 & 2 \\
\hline Total & 121 & 100 \\
\hline
\end{tabular}


members. The family caregivers' educational levels were as follows: elementary school $(\mathrm{N}=42,53 \%)$, high school $(\mathrm{N}=26,33 \%)$, and university graduates $(\mathrm{N}=9,12 \%)$. Two family caregivers were illiterate $(2 \%)$. Of the 82 family caregivers, 40 reported the presence of pain in their children (49\%), consistent with the children's/adolescents' report ( $N$ $=20)$ and the direct observation of pain signs $(N=20)$. The primary sign of pain observed by the family caregivers was crying behavior $(\mathrm{N}=14)$, which could be associated with the inpatients' facial or body expression. Of the 40 family caregivers who perceived pain, 19 (48\%) communicated their observations to the health professional team.

As seen in Figure 1, pain was reported by the inpatients and family caregivers using descriptors of the intensity and quality of the pain. Of all respondents, only $11 \%$ of the children/adolescents and $14 \%$ of the family caregivers did not describe the pain symptoms.

With regard to pain management, of the 20 children/ adolescents who reported pain, 13 additionally reported a successful pain relief intervention, whereas 7 did not perceive any pain management. Furthermore, of the 40 family caregivers who detected pain in their children, 28 (70\%) observed successful pain management. Pharmacological prescriptions were the primary pain interventions perceived by both inpatients and their family caregivers.

\section{Pediatric pain reports: health professionals}

Both physicians and nurses primarily detected acute pain associated with medical procedures. The physicians reported pain in 44 inpatients (38\%), which was acute in 41 (93\%). Physicians associated pain with three main events: clinical procedures ( $N=26,59 \%$ of patients), clinical evolu- tion of a disease ( $\mathrm{N}=9,20 \%$ of patients), and post-surgical recovery/rehabilitation ( $\mathrm{N}=5,11 \%$ of patients). The nurses reported pain in 60 inpatients $(50 \%)$, which was acute in $92 \%(\mathrm{~N}=55)$. The primary contexts, which the nurses predominantly associated with pain, were clinical procedures ( $\mathrm{N}=41,68 \%$ of patients) and the clinical evolution of a disease ( $N=9,15 \%$ of patients).

The primary clinical procedures that the health professionals perceived as causing pain were blood collection, mechanical ventilation, and physical examination (Figure 2).

As seen in Figure 3, both types of health professionals detected pain located predominantly in the limbs (physicians $=22 \%$ and nurses $=37 \%$ ), abdominal $/$ back region (physicians $=24 \%$ and nurses $=15 \%$ ), and face, head and neck (physicians $=19 \%$ and nurses $=15 \%$ ).

Systematic pain assessments were not mentioned by the health professionals, with the exception of $2 \%$ of physicians who did report measurements of pain. Physicians assessed pain based on the inpatients' self-reports (in 51\% of patients) and clinical examinations (in $47 \%$ of patients), whereas nurses predominately assessed pain signs using clinical observations (in $85 \%$ of patients). The major pain sign observed by both types of health professionals was clinical functional change (physicians $=78 \%$ of patients; nurses $=52 \%$ of patients). In addition, the nurses perceived crying as a pain sign in $35 \%$ of the patients. Facial activation, which is a validated behavioral pain sign in neonates, was less often reported by professionals (physicians $=7 \%$ of patients; nurses $=9 \%$ of patients).

For the 44 inpatients whose pain was detected by the physicians, pain was successfully managed in $66 \%$, and

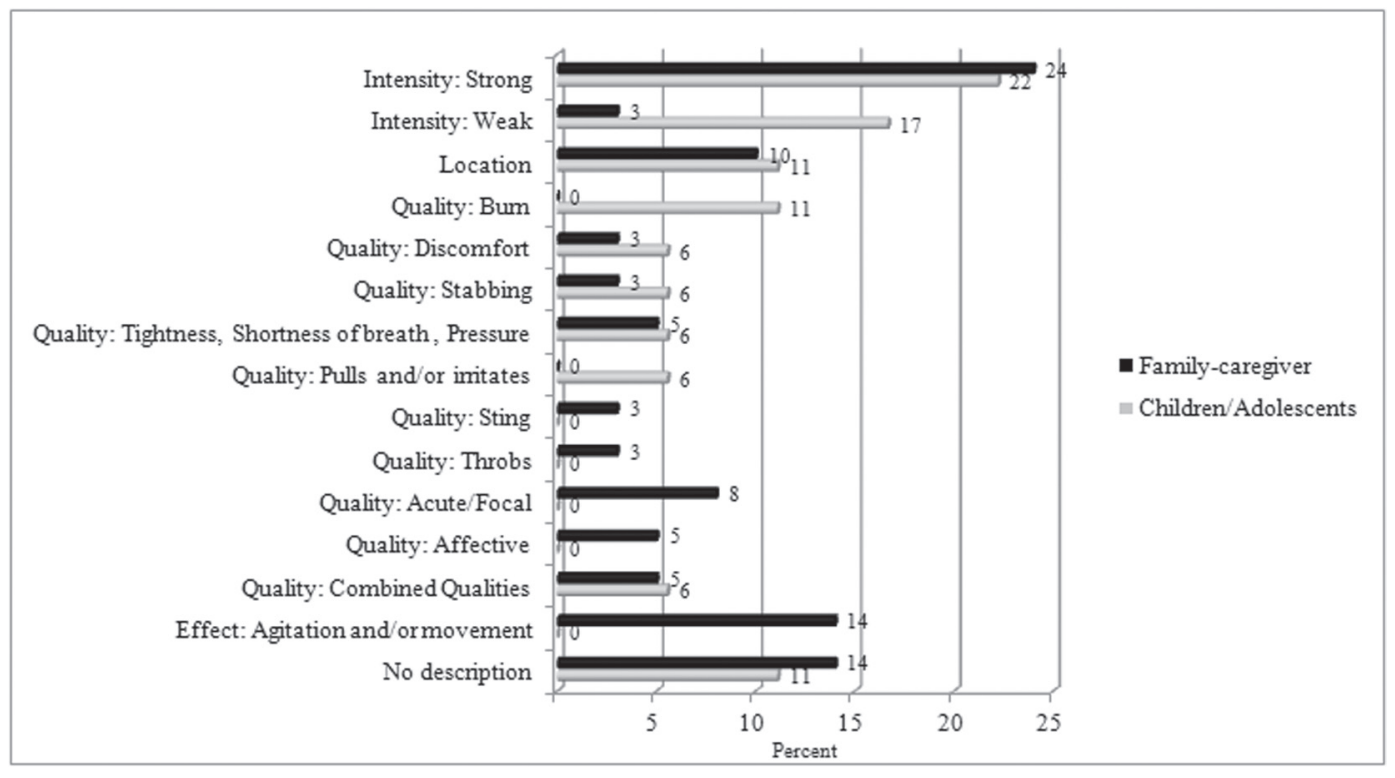

Figure 1. Child/adolescent inpatients' self-reports and family caregivers' hetero-reports about pain type. Child/ adolescent inpatients: $\mathrm{N}=18$, missing data $=2$; family caregivers: $\mathrm{N}=37$, missing data $=3$. 


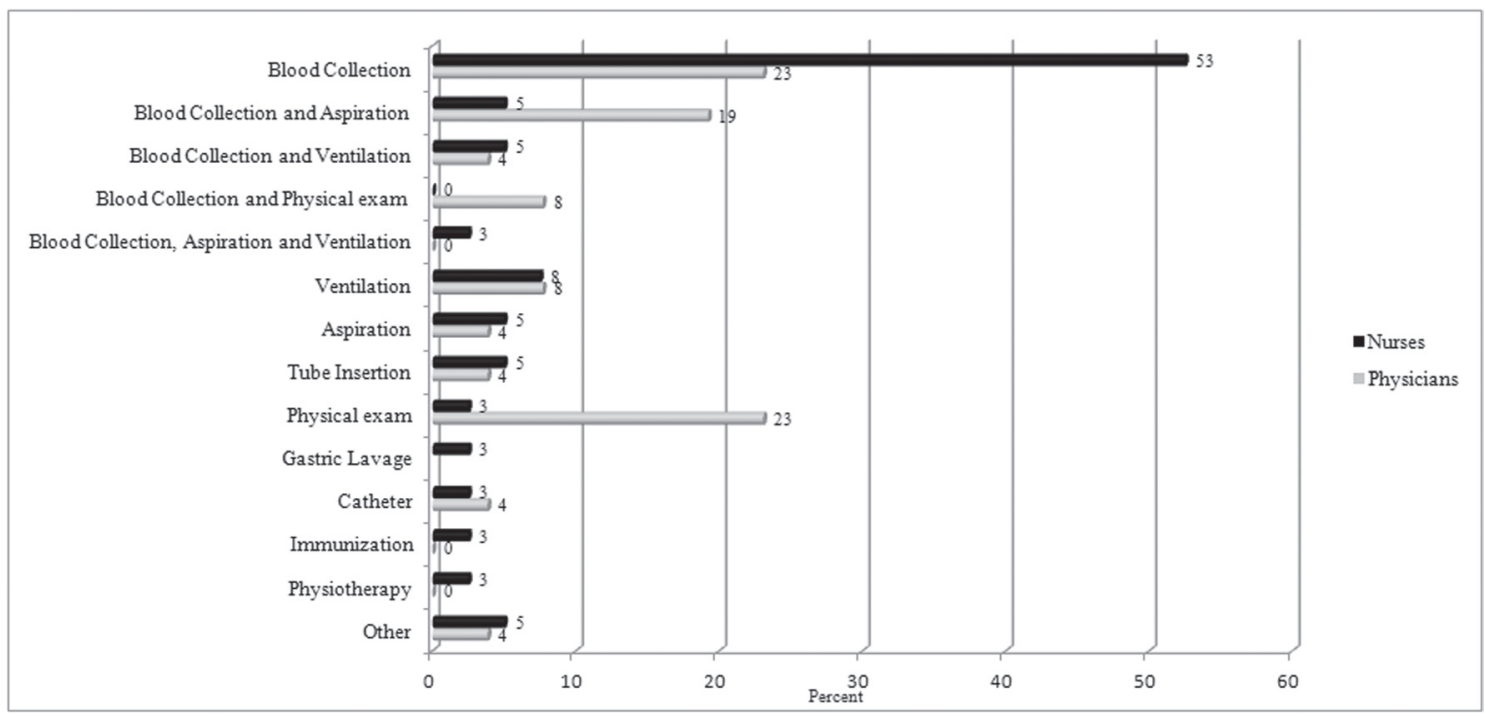

Figure 2. Clinical procedures in which the health professionals detected pediatric pain. Physicians: $N=26 ; n u r s e s: ~ N=$ 42, missing data $=1$.

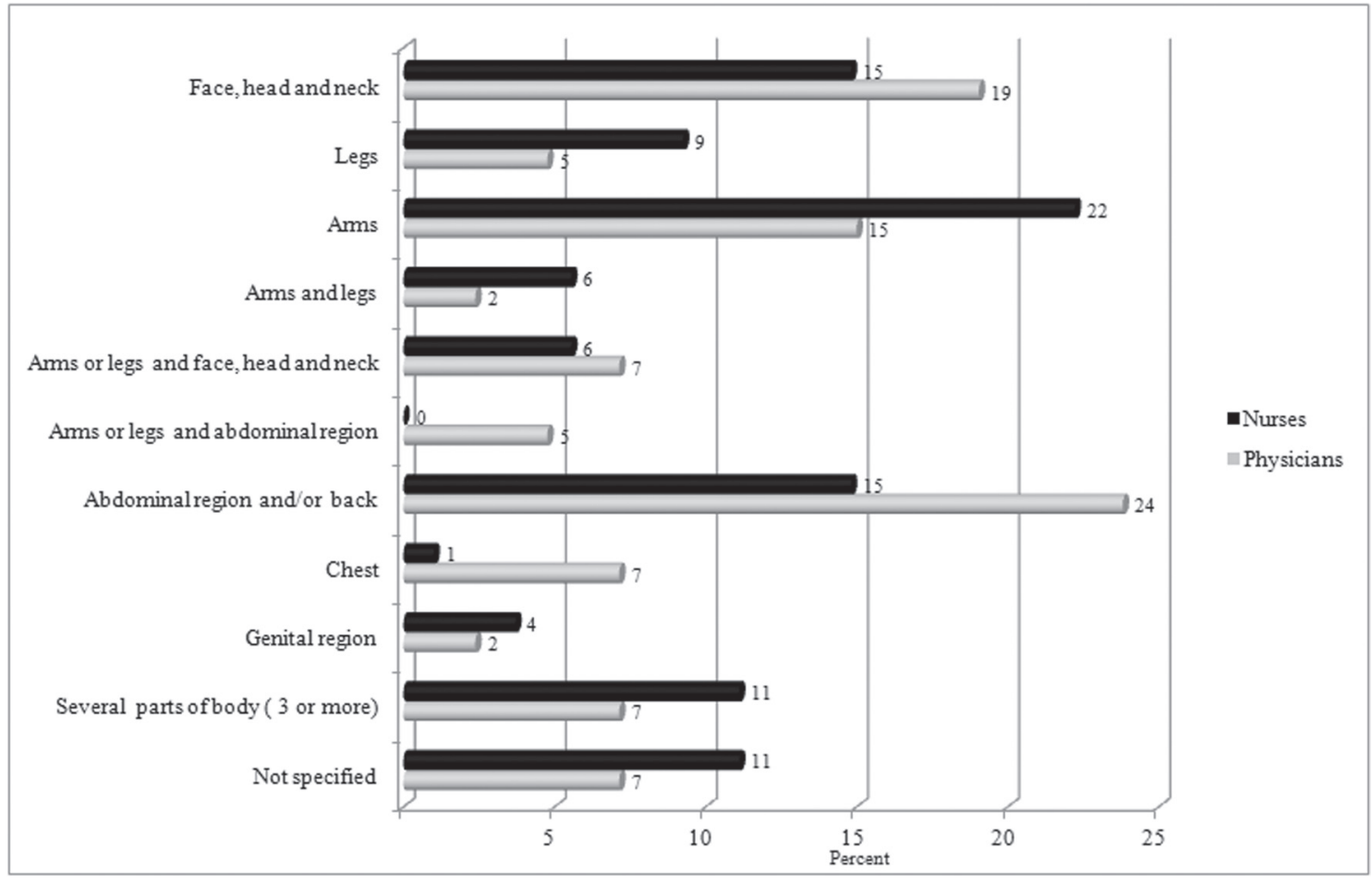

Figure 3. Pain localization according to health professionals' observations. Physicians: $N=44$, missing data $=2$; nurses: $\mathrm{N}=60$, missing data $=6$.

pharmacological management (analgesic) was adopted in $79 \%$ of treated cases. Non-pharmacological interventions were less often reported by physicians. In addition, they identified the use of sucrose for pain relief as nonpharmacological management.
Among 60 inpatients whose pain was detected by the nurses, pain was successfully managed in $78 \%$, and pharmacological management (analgesic) was used in 68\% of treated cases. Non-pharmacological interventions were reported by nurses in $49 \%$ of these patients. They identi- 
fied the use of sucrose for $35 \%$ of the inpatients, and they similarly identified this substance as a non-pharmacological management.

\section{Medical/nursing chart records}

Medical and nursing charts were analyzed for 118 (97\%) of the 121 patients. No notations were found regarding pain in the majority of charts $(74 \% ; \mathrm{N}=87)$, even for inpatients with pain symptoms. Only 31 charts included an assessment of pain. Of these, 15 charts presented pain scores obtained by means of validated pain tools (Neonatal Infant Pain Scale, Neonatal Facial Coding System, and COMFORT Scale). These scales were more often recorded by the nurses $(\mathrm{N}=$ 13) than by the physicians $(N=2)$.

Records of pharmacological management, including analgesic prescriptions alone ( $\mathrm{N}=56,90 \%)$ or associated with sedatives ( $N=6,10 \%)$, were found in 62 medical charts (51\%). Of these 62 charts, $44 \%$ were found to include pain relief prescriptions and administration records, $29 \%$ of the charts included the medicines prescribed but not administered to the patients, and $27 \%$ had records of prescriptions; however, no information was recorded concerning the effectiveness of their administration.

\section{Associations of pain reports by inpatients/family caregivers, pain perceptions of the health professionals, and pain recordings}

Regarding the medical charts, there were statistically significant associations between the pain reports of children/ family-caregivers and the following variables: the recording of the presence of pain in the medical/nurse charts $\left(X^{2}=16.21\right.$; $P \leq 0.00001)$, and also the recording of pain management strategies $\left(X^{2}=5.46 ; P \leq 0.019\right)$. In addition, analysis of the associations showed that the pain reports of inpatients or their family-caregivers and health professionals (physicians and nurses) agreed significantly in 59 cases $\left(68 \% ; x^{2}=\right.$ 11.75; $P \leq 0.001)$.

\section{Discussion}

In the present study, regarding the prevalence of pain in the $24 \mathrm{~h}$ prior to the target day for data collection, $59 \%$ of 34 children/adolescents who were able to verbalize presented pain complaints. Additionally, $49 \%$ of 82 family caregivers recognized their children's pain signs, and the majority reported their observations to the health professional team. The physicians reported pain in $38 \%$ of inpatients, and the nurses reported pain in $50 \%$.

These results are similar to previous findings that demonstrated, on the one hand, the high prevalence of pediatric pain and, on the other hand, the under-recognition and undertreatment of pain in hospitalized children (1-4,14). In the study of Taylor et al. (3), the prevalence of pediatric pain was $64 \%$ in the $24 \mathrm{~h}$ prior to the interview. In addition, Karling et al. (1) identified that, even under treatment, moderate to severe pain occurred in $23 \%$ of inpatients during the postoperative period and in $31 \%$ of patients with pain of another origin.

Stevens et al. (4) assessed pain epidemiological and management procedures through a review of medical charts from Canadian hospitals. In that study, children had undergone at least one painful procedure in the 24-h period preceding data collection (mean: 6.3 per child), and $84.8 \%$ had a pharmacological intervention. However, the records of only $28.3 \%$ of children mentioned one or more pain management intervention administered and documented specifically for a painful procedure.

Regarding the self-report of pain, in the present study $90 \%$ of children older than 24 months reported their pain symptoms, primarily to family caregivers. The children described their pain symptoms precisely, using dimensions of quality, intensity, and localization. This result confirms the previous study of Stanford et al. (15), in which children of 18 months of age were cognitively competent to communicate pain symptoms using spontaneous verbal descriptors. Considering that pain is a subjective phenomenon, it is very important to obtain a self-report of the child's pain, and such a report may be accessible from young, preschool-aged children.

As we expected, in the current study, non-verbal infants less than 24 months of age were absolutely dependent on caregivers to detect their pain symptoms. The family caregivers identified their child's pain primarily through crying behaviors, and $49 \%$ of family caregivers communicated their observations to the health professional team.

As pointed out by Anand et al. (16), pain evaluation in this especially vulnerable population is a challenge for health professional teams. Pain can be experienced by infants despite their inability to verbally communicate that experience (17). Repeated pain experiences suffered in the early stages of development by these vulnerable infants could provoke negative consequences in later childhood $(18,19)$.

Pain assessment in this population should be adapted to focus on behavioral, physiological, and contextual parameters. Typically, these indicators are included in validated pain tools for infant patients such as the Neonatal Infant Pain Scale/NIPS, the Neonatal Facial Coding System/NFCS, the Premature Infant Pain Profile/PIPP, and the COMFORT scale (20). Well-established instruments for pediatric pain assessment can be found in PED-IMMPACT Task Force reviews of observational procedures (21) and report measures (22).

The results of the present study show differences in the perception of children's pain by health professionals. Nurses identified the presence of children's pain in $50 \%$ of cases in the $24 \mathrm{~h}$ preceding data collection more frequently than did the physicians (38\%). A study by Pillai Riddell and Craig (23) examined differences in the judgments of pediatric pain among different infant caregiver groups. They found that the caregivers exhibited important differences in how they perceived infants' pain. Pediatricians attributed lower levels of pain than parents, whereas nurses were intermediate to the other groups and did not differ significantly from either group. 
These differences in the caregivers' perceptions of pain could inhibit pain management and contribute to the unnecessary suffering of children. Therefore, in order to be effective, the assessment of pain must be made using multiple, mixed methods including self- and hetero-reporting, behavior and biological indicators, and contextual indicators.

Our characterization of pain assessment revealed that this procedure is not routinely performed in the hospital setting, given that $74 \%$ of the charts lacked a record of pain. In addition, only 15 of 118 charts included a pain record using a validated pain scale measurement. Taylor et al. (3) reviewed patient charts and showed similar findings: $73 \%$ of the records they reviewed had no pain score documented during the preceding $24 \mathrm{~h}$. Similarly, Karling et al. (1) found that pain assessment in children was not performed regularly in the majority of departments of Swedish hospitals, and validated measurements were used infrequently. Those findings reflect a deficit in the transfer of scientific knowledge to clinical practice despite many validated pain measurements available in the literature. Pain records were not well documented in the medical charts because there were no systematic and regular recordings of pain assessment and management, especially regarding non-pharmacological interventions.

Despite the available evidence about the effectiveness of pharmacological and non-pharmacological interventions for children's pain relief, the present study suggests that pain management remains inadequate. On the one hand, pharmacological interventions were predominantly used in all pediatric wards of the hospital studied. On the other hand, non-pharmacological interventions were less frequently noted than pharmacological interventions by health professionals. The use of sucrose in neonates and infants caused some misunderstandings because the substance was included as both a non-pharmacological and a pharmacological intervention by the professional teams. Other non-pharmacological interventions, such as breastfeeding, non-nutritive sucking, distraction, and relaxation, were identified infrequently. This infrequent reference to the use of other non-pharmacological interventions could either reflect a lack of knowledge about this modality of intervention or the fact that the health professionals did not recognize these as pain management techniques.

Finally, protocols, guidelines, and policies regarding pain assessment and management were not identified by professionals who cared for pediatric inpatients. Even if such policies were implemented in clinical services, the physicians and nurses did not recognize them as institutional initiatives. Harrison et al. (2) demonstrated that the majority of Australian Neonatal Units rarely used current evidence-based strategies to reduce procedural pain in hospitalized infants because they had no articulated policy to guide pain management.

The present study adds to the literature about pediatric pain in developing countries and, unfortunately, confirms similar results previously found in pain studies carried out in developed countries. The findings also demonstrate the under-recognition and under-treatment of pain in a pediatric inpatient population. As far as we know, this is the first pain survey study focusing on all pediatric clinical services in a Brazilian public teaching hospital setting. The data were obtained from mixed sources of information including the reports of pediatric inpatients, family caregivers, nurses and physicians, and the records of medical/nursing charts. This methodology offered a complete picture from multiple perspectives of the phenomenon of pain, including current and direct information about pain, and did not, therefore, rely only on the retrospective analysis of medical charts.

However, some limitations should be acknowledged. First, the data are a "snapshot", and as such are restricted to one specific day. Second, the data are based on reports and did not include direct observation of the clinical routines to better evaluate pain assessment and management. Third, the sample was small, but included all the patients hospitalized on the pediatric wards on the target day of data collection. To reach a broad generalization of the findings of the present study, new studies should be addressed in other hospitals with characteristics similar to those of our study. As a consequence of the current study, seven public hospitals in Brazil have been replicating this investigation; similar studies are in progress using the same methodology as the present one, aiming to evaluate prevalence, assessment and management of pain in child /adolescent inpatients.

In conclusion, as Pang et al. (24) wrote: "It is astonishing that in 21 st century decisions on health care can still be made without a solid grounding in research evidence". Typically, the literature offers updated, evidence-based, efficacy procedures for pediatric pain assessment and management. The challenge is to unite researchers, clinicians, and policy makers in achieving the shared goal of reducing or eliminating unnecessary pain experiences and adequately managing the inevitable pain in a pediatric population.

\section{Acknowledgments}

The authors are grateful for the financial support of Global Health Research Initiative - Canadian Institutes of Health Research (CIHR), and the special collaboration of the administration of the Hospital and the directors of the clinical and surgery services. In addition, we recognize the relevant support of Pain in Child Health $(\mathrm{PICH})$, a strategic research training initiative of the CIHR. We also are very thankful to the patients and their families and to the health professionals for participating in the study.

\section{Supplementary material}

Interview guides 


\section{References}

1. Karling M, Renstrom M, Ljungman G. Acute and postoperative pain in children: a Swedish nationwide survey. Acta Paediatr 2002; 91: 660-666.

2. Harrison D, Loughnan $P$, Johnston L. Pain assessment and procedural pain management practices in neonatal units in Australia. J Paediatr Child Health 2006; 42: 6-9.

3. Taylor EM, Boyer K, Campbell FA. Pain in hospitalized children: a prospective cross-sectional survey of pain prevalence, intensity, assessment and management in a Canadian pediatric teaching hospital. Pain Res Manag 2008; 13: 25-32.

4. Stevens BJ, Abbott LK, Yamada J, Harrison D, Stinson J, Taddio A, et al. Epidemiology and management of painful procedures in children in Canadian hospitals. CMAJ 2011; 183: E403-E410.

5. Melotti RM, Samolsky-Dekel BG, Ricchi E, Chiari P, Di Giacinto I, Carosi F, et al. Pain prevalence and predictors among inpatients in a major Italian teaching hospital. A baseline survey towards a pain free hospital. Eur J Pain 2005; 9: 485-495.

6. Schechter NL, Finley GA, Bright NS, Laycock M, Forgeron P. ChildKind: a global initiative to reduce pain in children. Ped Pain Letter 2010; 12: 26-30.

7. Elias LS, Guinsburg R, Peres CA, Balda RC, Santos AM. Disagreement between parents and health professionals regarding pain intensity in critically ill neonates. J Pediatr 2008; 84: 35-40

8. Chermont AG, Guinsburg R, Balda RC, Kopelman BI. [What do pediatricians know about pain assessment and treatment in newborn infants?]. J Pediatr 2003; 79: 265-272.

9. Prestes AC, Guinsburg R, Balda RC, Marba ST, Rugolo LM, Pachi PR, et al. [The frequency of pharmacological pain relief in university neonatal intensive care units]. J Pediatr 2005; 81: 405-410.

10. Kulkamp IC, Barbosa CG, Bianchini KC. [The perception of health professionals about pain management and opioid use: a qualitative study]. Cien Saude Colet 2008; 13 (Suppl): 721-731.

11. Daudt AW, Hadlich E, Facin MA, Aprato RM, Pereira RP. [Opiates in pain management: correct or underestimated use? Data from a university hospital]. Rev Assoc Med Bras 1998; 44: 106-110.

12. Lerner RB, Carvalho M, Vieira AA, Lopes JM, Moreira ME. Medication errors in a neonatal intensive care unit. $J$ Pediatr 2008; 84: 166-170.
13. Schechter NL, Finley GA. The ChildKind Initiative: a program to reduce pain in child health facilities worldwide [ChildKind Web Site]. http://www.childkindinternational.org/ChildKind_ International/Resources.html. Accessed May 24, 2010.

14. Carbajal R, Rousset A, Danan C, Coquery S, Nolent P, Ducrocq $S$, et al. Epidemiology and treatment of painful procedures in neonates in intensive care units. JAMA 2008; 300: 60-70.

15. Stanford EA, Chambers CT, Craig KD. A normative analysis of the development of pain-related vocabulary in children. Pain 2005; 114: 278-284.

16. Anand KJ, Aranda JV, Berde CB, Buckman S, Capparelli $\mathrm{EV}$, Carlo W, et al. Summary proceedings from the neonatal pain-control group. Pediatrics 2006; 117: S9-S22.

17. Stevens BJ, Anand KJS, McGrath PJ. An overview of pain in neonates and infants. In: Anand KJS, Stevens BJ, McGrath PJ (Editors), Pain in neonates and infants. Toronto: Elsevier; 2007. p 1-9.

18. Grunau RE, Tu MT. Long-term consequences of pain in human neonates. In: Anand KJS, Stevens BJ, McGrath PJ (Editors), Pain in neonates and infants. Toronto: Elsevier; 2007. p 45-55.

19. Klein VC, Gaspardo CM, Martinez FE, Grunau RE, Linhares MB. Pain and distress reactivity and recovery as early predictors of temperament in toddlers born preterm. Early Hum Dev 2009; 85: 569-576.

20. Stevens BJ, Pillai-Riddell RR, Oberland TE, Gibbina S. Assessment of pain in neonates and infants. In: Anand KJS, Stevens BJ, McGrath PJ (Editors), Pain in neonates and infants. Toronto: Elsevier; 2007. p 67-90.

21. von Baeyer CL, Spagrud LJ. Systematic review of observational (behavioral) measures of pain for children and adolescents aged 3 to 18 years. Pain 2007; 127: 140-150.

22. Stinson JN, Kavanagh T, Yamada J, Gill N, Stevens B. Systematic review of the psychometric properties, interpretability and feasibility of self-report pain intensity measures for use in clinical trials in children and adolescents. Pain 2006; 125: 143-157.

23. Pillai Riddell RR, Craig KD. Judgments of infant pain: the impact of caregiver identity and infant age. J Pediatr Psychol 2007; 32: 501-511.

24. Pang T, Terry RF, The PLoS Medicine Editors. WHO/PLoS collection "No health without research": a call for papers. PLoS Med 2011; 8: e1001-e1008. 